\title{
Qualidade de Vida no Trabalho e nível de estresse dos profissionais da atenção primária
}

\author{
Quality of Working Life and stress level of professionals in Primary \\ Health Care
}

Geovane Krüger Moreira de Lima', Ludmila Mourão Xavier Gomes', Thiago Luis de Andrade

Barbosa'

DOI: $10.1590 / 0103-1104202012614$

RESUMO Estudo com objetivo de avaliar a Qualidade de Vida no Trabalho (QVT) e o nível de estresse de trabalhadores da Atenção Primária à Saúde (APS) em uma cidade de tríplice fronteira. Trata-se de pesquisa transversal da qual participaram 120 profissionais da APS, que responderam a questionários sociodemográfico, ocupacional, de hábitos de vida, instrumento de QVT abreviado (QWLQ-Bref) e Escala do Estresse Percebido (PSS 13). Verificou-se associação da QVT e dos níveis de estresse com variáveis sociodemográficas e de estilo de vida com uso do teste t de Student, Anova, Mann-Whitney e KruskalWallis. Em seguida, realizou-se regressão linear múltipla. Os resultados mostraram escore médio de QVT dos profissionais de $62,8 \pm 10,0$ e escore médio de estresse percebido de 24,5 $\pm 6,0$. A variável satisfação com o trabalho se manteve estatisticamente associada à QVT global, com o domínio psicológico e o nível de estresse. A cor da pele e o sexo se associaram aos domínios pessoal e profissional da QVT, respectivamente. Conclui-se que a satisfação com o trabalho é um fator importante na determinação da melhor QVT e na proteção contra o estresse. Esses resultados apontam aspectos a serem considerados pelos serviços de saúde para melhor abordagem à saúde mental do trabalhador.

PALAVRAS-CHAVE Qualidade de vida. Estresse psicológico. Saúde do trabalhador. Atenção Primária à Saúde. Pessoal de saúde.

\begin{abstract}
This study aimed to evaluate the Quality of Working Life ( $Q W L)$ and stress level of professionals of Primary Health Care (PHC) in a city of triple border. This is a cross-sectional study in which 120 professionals of $P H C$ participated, and answered socio-demographic, occupational, lifestyle, abbreviated QWL (QWLQ-Bref) and Perceived Stress Scale (PSS 13) questionnaires. It was verified association between QWL and stress levels with sociodemographic and lifestyle variables through the Student's t-test, Anova, Mann-Whitney and Kruskal-Wallis. Then, multiple linear regression model was performed. The results showed average professional QWL assessment of $62.8 \pm 10.0$ and average total perceived stress score of the participants was $24.5 \pm 6.0$. The variable satisfaction with work remained statistically associated with global QLW, with the psychological domain and the level of stress. Skin color and sex are associated with personal and professional domains, respectively. It is concluded that work satisfaction is an important factor in determining the best QWL and protection against stress. These results point out aspects to be considered by the health services to better approach the worker's mental health.
\end{abstract}

1 Universidade Federal da Integração LatinoAmericana (Unila) - Foz do Iguaçu (PR), Brasil. Igvictor@oi.com.br 


\section{Introdução}

Os trabalhadores da saúde têm sido alvo de inúmeras situações produtoras de estresse na execução da atividade laboral que afetam diretamente sua qualidade de vida. Sabe-se que um dos campos de atuação do Sistema Único de Saúde (SUS) é o da saúde do trabalhador, por meio da Política Nacional de Segurança e Saúde do Trabalhador ${ }^{1}$, que busca, sobretudo, propiciar ambientes saudáveis de trabalho. As condições e a organização do trabalho estão ligadas tanto à promoção quanto à Qualidade de Vida no Trabalho (QVT) 2,3 e, também, à redução de fatores propulsores do estresse. A QVT apresenta-se como fenômeno multifatorial relacionado com aspectos físicos, ambientais e psicológicos do ambiente de trabalho ${ }^{4}$.

Com a proposta de organização dos serviços de saúde em redes de atenção à saúde, destacam-se os trabalhadores que atuam na Atenção Primária à Saúde (APS), que se constitui em porta de entrada e ordenadora do cuidado. Existe o entendimento de que esses trabalhadores são elementos estratégicos na qualidade do cuidado prestado à população, bem como na efetivação das políticas de saúde. Entretanto, os locais onde se desenvolvem os processos de trabalho ainda se configuram como ambientes em frequentes tensões para as equipes de saúde e para os usuários ${ }^{5}$.

Com a expansão da Estratégia Saúde da Família (ESF) como modelo de reorganização da APS no Brasil, sobretudo com as particularidades próprias ao seu funcionamento em grandes cidades, crescem cada vez mais as insatisfações no trabalho e reivindicações das equipes de saúde ${ }^{6}$. Nessa perspectiva, a qualidade de vida no ambiente profissional permanece como elemento preocupante no desempenho do trabalho em saúde.

O processo de trabalho da equipe de saúde da família encontra-se direcionado por duas vertentes: uma é a gestão, que exige efetivação de metas e produção; e a outra é o usuário do SUS, que exige melhores condições nos cuidados de saúde prestados ${ }^{7,8}$. Contudo, a realidade encontrada nos serviços de saúde aponta algo difícil de ser conquistado pelos profissionais, segundo essas vertentes. Estudo realizado com trabalhadores da APS no Brasil constatou associação entre condições de trabalho psicossociais adversas e má qualidade de vida de trabalhadores, necessitando, dessa maneira, de ações intersetoriais destinadas a prosperar a saúde e a QVT 9 .

Outra questão importante que se relaciona com a QVT é o estresse laboral sofrido pelos profissionais da APS diante de tantas demandas de trabalho, predispondo a sintomas físicos e psicológicos, como ansiedade, depressão e síndrome de burnout. Ao se considerar o estresse como problema de ampla discussão nos dias atuais, cresce o interesse por compreender melhor os fatores que se refletem na saúde do trabalhador. Nesse sentido, é imprescindível discutir os fatores de riscos psicossociais e ambientais relacionados ao trabalho geradores do estresse laboral. Essas questões são percebidas nas relações sociais no trabalho e estão ligadas à competitividade/produtividade e à qualidade do produto, e devem ser analisadas à luz da qualidade do trabalho e em defesa da vida e da saúde dos trabalhadores ${ }^{10}$.

Nesse contexto, frente à escassez de dados sobre a temática no País, faz-se necessário conhecer a realidade da QVT e o nível de estresse dos trabalhadores da APS, no sentido de prover meios para melhorar o desempenho profissional das equipes, materializados em ações de promoção e prevenção. Diante do exposto, este estudo teve por objetivo avaliar a QVT e o nível de estresse de trabalhadores da APS em uma cidade de tríplice fronteira.

\section{Material e métodos}

Trata-se de estudo transversal conduzido com profissionais da APS do distrito sanitário leste de Foz do Iguaçu (PR), no período de março a dezembro de 2018. O município está situado no extremo oeste do estado do Paraná e faz fronteira com Paraguai e Argentina. Possui 
população de 264.044 habitantes, destacando-se pelo turismo, comércio e alto fluxo de pessoas em região de tríplice fronteira ${ }^{11}$. O distrito sanitário leste apresenta 06 Unidades Básicas de Saúde (UBS), sendo 03 no modelo tradicional e 03 na modalidade de Saúde da Família, contando com 09 equipes de saúde e 01 Núcleo Ampliado de Saúde da Família e Atenção Básica (Nasf-AB).

Primeiramente, a proposta da pesquisa foi apresentada à Secretaria Municipal de Saúde de Foz do Iguaçu (PR), e, em seguida, foi realizado um primeiro contato com a gerência das UBS para que os instrumentos de coleta de dados fossem aplicados em horários adequados à demanda de cada profissional, e assim não prejudicassem o processo laboral. Os participantes foram informados previamente da pesquisa durante as reuniões de equipe, realizadas semanalmente nas UBS.

Foram convidados a participar todos os profissionais atuantes nesse distrito sanitário do município. A amostra contou com profissionais das seguintes categorias: médico, enfermeiro, técnico de enfermagem, auxiliar de enfermagem, agente comunitário de saúde, dentista, auxiliar de saúde bucal, farmacêutico, fisioterapeuta, nutricionista, psicólogo, assistente social, recepcionista, gerente de UBS e auxiliar de serviços gerais.

Os critérios de inclusão da pesquisa foram: profissionais atuantes na APS do distrito sanitário leste do município que estavam exercendo suas funções há, pelo menos, um ano. Foram excluídos do estudo: profissionais que estavam em suas funções há menos de um ano, os que estavam afastados das suas funções laborais por motivo de doença ou de férias no período da pesquisa, os ausentes após três tentativas da coleta de dados, assim como os que não aceitaram participar da pesquisa.

Para cada profissional, foram informados os objetivos da pesquisa e que os dados obtidos seriam utilizados exclusivamente para fins científicos, assegurando, assim, a confidencialidade, o sigilo e o anonimato do sujeito participante. A participação se deu mediante assinatura do Termo de Consentimento Livre e Esclarecido.

No estudo, foram aplicados três instrumentos para investigação proposta: questionário sociodemográfico, ocupacional e hábitos de vida, o Questionário de Qualidade de Vida no Trabalho versão abreviada denominado Quality of Working Life Questionnaire (QWLQ-bref) e a Escala de Estresse Percebido com 13 itens (Perceived Stress Scale - PSS 13).

Para a coleta de dados sobre o perfil sociodemográfico, ocupacional e de hábitos de vida, empregou-se questionário construído com base na revisão de literatura. Nesse instrumento, foram avaliadas as seguintes variáveis: sexo (masculino/feminino), idade (até 45 anos/maior que 45 anos), estado civil (com companheiro/sem companheiro), cor da pele (branco/não branco), número de filhos (até 02/ mais que 02), nível de formação (fundamental/ médio ou superior), tempo de serviço na APS (até 11 anos/maior que 11 anos), renda familiar em Salários Mínimos (SM): (até 03 SM/04 a 07 $\mathrm{SM} / 08$ ou mais SM), tipo de moradia (própria/ alugada-cedida), realiza atividade física ( $\mathrm{sim} /$ não), tabagista (sim/não), autopercepção da alimentação (positiva/negativa), comorbidade autorreferida (sim/não), uso de medicação contínua (sim/não), dor autorreferida (sim/ não), dor autorreferida relacionada ao trabalho (sim/não), satisfação com o trabalho (satisfeito/insatisfeito) e afastamento prévio do trabalho devido à dor (sim/não).

Com o intuito de mensurar a QVT, sob o ponto de vista pessoal, de saúde, psicológico e profissional, aplicou-se questionário QWLQ-bref, constituído de 20 questões das 78 que constituem o QWLQ-78, versão completa do instrumento. O QWLQ-bref apresenta quatro questões do domínio físico/ saúde, três do domínio psicológico, quatro do domínio pessoal e nove do domínio profissional12. As respostas do instrumento seguem a escala tipo likert com a classificação: 1-nada, 2-muito pouco, 3-mais ou menos, 4-bastante, 5 -extremamente. O escore global de avaliação do QWLQ-bref possui escala de 0 a 100 
e apresenta a seguinte classificação: muito insatisfatório ( 0 a 22,5), insatisfatório (22,5 a 45 ), neutro ( 45 a 55 ), satisfatório ( 55 a 77,5$)$, muito satisfatório (77,5 a 100).

O nível de estresse foi mensurado pelo PSS 13 de Cohen, Kamarck e Mermelstein ${ }^{\mathbf{1 3}}$, que contém 13 itens relacionados ao trabalho e que se referem à frequência com que o entrevistado se sentiu ou pensou durante o último mês que precedeu a data da entrevista. As respostas do instrumento seguem a escala tipo likert com a classificação: 0-nunca, 1-quase nunca, 2-algumas vezes, 3-com muita frequência, 4 -muitas vezes. No instrumento, os itens 04, 05, 06, 07, 09 e 10 apresentam pontuação invertida. $\mathrm{O}$ escore total do PSS 13 varia entre zero e 52 pontos.

Foi realizado estudo piloto prévio com 10 profissionais da APS que trabalhavam em outros distritos sanitários e que, portanto, não entraram na análise dos dados desta pesquisa. Os profissionais não relataram qualquer dificuldade ou dúvida no preenchimento do questionário, o qual foi mantido inalterado para o estudo principal.

Os dados foram tabulados no programa Microsoft Excel versão 2016 e, posteriormente, avaliados utilizando-se o pacote estatístico Bioestat 5.0, da Universidade Federal do Pará (UFPA). Foi realizada análise bivariada para verificar associação da QVT e dos níveis de estresse com variáveis sociodemográficas, ocupacionais e de hábitos de vida. Para a determinação do teste estatístico, foi verificada a normalidade de distribuição das variáveis através do teste de Kolmogorov-Smirnov. Nas variáveis que apresentaram distribuição normal, aplicaram-se os testes t de Student para amostras independentes e Anova. Para as variáveis com distribuição não paramétrica, utilizaram-se os testes Mann-Whitney e Kruskal-Wallis. O nível de significância adotado foi de $5 \%$.

Durante a análise dos dados, buscou-se identificar as variáveis que poderiam estar relacionadas à QVT e aos níveis de estresse percebido dos profissionais atuantes nas UBS em modelos explicativos. Inicialmente, consideraram-se os resultados da análise bivariada, tomando-se todas as variáveis com $\mathrm{p}<0,20$. Em seguida, avaliou-se a QVT e o estresse percebido como variável dependente, mediante o emprego de modelos de regressão linear múltipla, em função de características sociodemográficas, ocupacionais e de hábitos de vida, para definir os modelos explicativos.

A investigação respeitou as normas éticas de pesquisa que envolve seres humanos da Resolução no 466/2012 do Conselho Nacional de Saúde/Ministério da Saúde, sendo aprovada pelo Comitê de Ética e Pesquisa da Universidade Estadual do Oeste do Paraná (Unioeste), sob parecer $\mathrm{n}^{\mathrm{0}} 2.515 .670 / 2018$, com CAAE nº 83164318800000107.

\section{Resultados}

Participaram do estudo a totalidade dos 120 profissionais da APS do distrito leste de Foz do Iguaçu (PR). Não houve perdas durante a coleta de dados. A tabela 1 apresenta a caracterização sociodemográfica, ocupacional e de hábito de vida dos participantes da pesquisa. Os dados apresentados revelaram maioria do sexo feminino (82,5\%), com indivíduos com idade até 45 anos (53,3\%), de cor da pele branca (61,7\%), que vivem com companheiro (82,5\%), com até dois filhos (81,7\%), com renda familiar de 04 a $07 \mathrm{SM}(70,8 \%)$ e que vivem em casa alugada ou cedida (55,0\%). Com relação ao nível de formação, a maioria possuía ensino fundamental/médio (79,2\%), com tempo de serviço na APS superior a 11 anos (70,8\%). No tocante aos hábitos de vida, $60,8 \%$ não realizam atividades físicas; 87,5\% não possuem hábito tabagista; $53,3 \%$ autopercebem a alimentação como negativa. A presença de comorbidade foi relatada por $51,7 \%$ dos profissionais; o uso de medicamento contínuo, por $50,8 \%$. A presença de dor foi referida por $52,5 \%$ dos profissionais; e a dor relacionada ao trabalho foi apontada por $42,5 \%$. Foi constatado que $63,3 \%$ dos pesquisados estão insatisfeitos com o trabalho; e a maioria (61,7\%) relatou não ter afastamento prévio do trabalho devido à dor. 
Tabela 1. Caracterização sociodemográfica, ocupacional e de hábitos de vida dos profissionais pesquisados da Atenção Primária à Saúde, distrito leste, Foz do Iguaçu (PR), 2018

\begin{tabular}{|c|c|c|}
\hline Variáveis & $\mathrm{n}$ & $\%$ \\
\hline \multicolumn{3}{|l|}{ Sexo } \\
\hline Feminino & 99 & 82,5 \\
\hline Masculino & 21 & 17,5 \\
\hline \multicolumn{3}{|l|}{ Idade } \\
\hline$\leq 45$ anos & 64 & 53,3 \\
\hline$>45$ anos & 56 & 46,7 \\
\hline \multicolumn{3}{|l|}{ Estado Civil } \\
\hline Com companheiro & 99 & 82,5 \\
\hline Sem companheiro & 21 & 17,5 \\
\hline \multicolumn{3}{|l|}{ Cor da pele } \\
\hline Branca & 74 & 61,7 \\
\hline Não Branca & 46 & 38,3 \\
\hline \multicolumn{3}{|l|}{ № de Filhos } \\
\hline$\leq 2$ & 98 & 81,7 \\
\hline$>2$ & 22 & 18,3 \\
\hline \multicolumn{3}{|l|}{ Nível de formação } \\
\hline Fundamental/Médio & 95 & 79,2 \\
\hline Superior & 25 & 20,8 \\
\hline \multicolumn{3}{|c|}{ Tempo Serviço na APS* } \\
\hline$\leq 11$ anos & 14 & 11,7 \\
\hline$>11$ anos & 85 & 70,8 \\
\hline \multicolumn{3}{|l|}{ Renda familiar } \\
\hline Até $03 \mathrm{SM}^{\star \star}$ & 14 & 11,7 \\
\hline 04 a 07 SM & 85 & 70,8 \\
\hline 08 ou mais SM & 21 & 17,5 \\
\hline \multicolumn{3}{|l|}{ Tipo de moradia } \\
\hline Própria & 54 & 45,0 \\
\hline Alugada/cedida & 66 & 55,0 \\
\hline \multicolumn{3}{|l|}{ Realiza atividade Física } \\
\hline Sim & 47 & 39,2 \\
\hline Não & 73 & 60,8 \\
\hline \multicolumn{3}{|l|}{ Tabagismo } \\
\hline $\operatorname{Sim}$ & 15 & 12,5 \\
\hline Não & 105 & 87,5 \\
\hline \multicolumn{3}{|c|}{ Autopercepção da alimentação } \\
\hline Positiva & 56 & 46,7 \\
\hline Negativa & 64 & 53,3 \\
\hline \multicolumn{3}{|c|}{ Comorbidade autorreferida } \\
\hline Sim & 58 & 48,3 \\
\hline Não & 62 & 51,7 \\
\hline \multicolumn{3}{|c|}{ Uso de medicamento contínuo } \\
\hline Sim & 61 & 50,8 \\
\hline Não & 59 & 49,2 \\
\hline \multicolumn{3}{|l|}{ Dor autorreferida } \\
\hline Sim & 63 & 52,5 \\
\hline Não & 57 & 47,5 \\
\hline
\end{tabular}


Tabela 1. (cont.)

\begin{tabular}{lcr}
\hline Variáveis & $\mathbf{n}$ & $\%$ \\
\hline Sim & 51 & 42,5 \\
Não & 69 & 57,5 \\
\hline Satisfeito com trabalho & & \\
\hline Satisfeito & 44 & 36,7 \\
Insatisfeito & 76 & 63,3 \\
\hline Afastamento do trabalho devido à dor & & 38,3 \\
\hline Sim & 46 & 61,7 \\
\hline Não & 74 &
\end{tabular}

*APS = Atenção Primária à Saúde. ${ }^{\star \star}$ SM = Salário Mínimo.

Neste estudo, a avaliação global média da QVT dos profissionais foi $62,8 \pm 10,0$, variando entre 34 e 91 pontos. A análise bivariada dos domínios físico, psicológico, pessoal e profissional e escore total da QVT dos profissionais e as variáveis estudadas estão apresentadas na tabela 2. Nessa análise, o escore total médio teve associação estatística com as variáveis sexo $(\mathrm{p}=0,016)$, com destaque para as mulheres $(66,8 \pm 7,3)$, idade $(\mathrm{p}=0,042)$ com maior média entre indivíduos com até 45 anos $(64,9 \pm 9,7)$, presença de dor $(p=0,029)$ sendo maior entre os profissionais que não relataram tal sintoma $(65,0 \pm 10,3)$, satisfação com o trabalho $(\mathrm{p}=0,002)$, com maior média entre aqueles que afirmaram estar satisfeitos $(66,5 \pm 9,0)$.

Tabela 2. Análise bivariada da QVT e variáveis sociodemográficas, ocupacionais e hábitos de vida dos profissionais da Atenção Primária à Saúde, distrito leste, Foz do Iguaçu (PR), 2018

\begin{tabular}{|c|c|c|c|c|c|}
\hline \multirow{2}{*}{$\begin{array}{l}\text { Domínio } \\
\text { Variáveis }\end{array}$} & \multicolumn{2}{|c|}{ Físico } & \multicolumn{3}{|c|}{ Psicológico } \\
\hline & Média & $\mathrm{DP}^{\star}$ & $p$ & Média & DP \\
\hline Sexo & & & 707 & & \\
\hline Masculino & 12,5 & 1,6 & & 10,1 & 1,5 \\
\hline Feminino & 12,2 & 2,0 & & 9,4 & 2,1 \\
\hline Idade & & & 953 & & \\
\hline$>45$ anos & 12,1 & 2,1 & & 9,2 & 2,2 \\
\hline$\leq 45$ anos & 12,4 & 1,8 & & 9,8 & 1,9 \\
\hline Estado Civil & & & 929 & & \\
\hline Com companheiro & 12,1 & 1,8 & & 9,6 & 1,8 \\
\hline Sem companheiro & 12,4 & 1,8 & & 9,2 & 2,0 \\
\hline Cor da pele & & & 761 & & \\
\hline Branca & 12,2 & 2,2 & & 9,7 & 2,2 \\
\hline Não Branca & 12,2 & 1,5 & & 9,1 & 1,8 \\
\hline № de Filhos & & & 852 & & \\
\hline$\leq 2$ & 12,2 & 2,0 & & 9,6 & 2,0 \\
\hline$>2$ & 12,3 & 1,8 & & 9,0 & 2,2 \\
\hline Nível de formação & & & 614 & & \\
\hline Fundamental/Médio & 12,2 & 1,9 & & 9,3 & 2,0 \\
\hline Superior & 12,4 & 2,0 & & 9,9 & 2,1 \\
\hline
\end{tabular}


Tabela 2. (cont.)

\begin{tabular}{|c|c|c|c|c|c|}
\hline Domínio & Fís & & & Psicol & \\
\hline Variáveis & Média & $\mathrm{DP}^{\star}$ & $\mathrm{p}$ & Média & DP \\
\hline Tempo Serviço na APS ${ }^{\star \star}$ & & & 66 & & \\
\hline$\leq 11$ anos & 12,3 & 1,9 & & 9,5 & 1,8 \\
\hline$>11$ anos & 12,1 & 2,0 & & 9,4 & 2,3 \\
\hline Renda familiar & & & 973 & & \\
\hline Até $3 \mathrm{SM}^{\star \star \star \star}$ & 12,4 & 2,2 & & 10,2 & 1,3 \\
\hline 04 a 07 SM & 12,3 & 1,8 & & 9,5 & 2,2 \\
\hline 08 ou mais SM & 11,9 & 2,4 & & 9,0 & 1,9 \\
\hline Tipo de moradia & & & 315 & & \\
\hline Própria & 12,2 & 1,9 & & 9,5 & 2,1 \\
\hline Alugada/cedida & 11,4 & 2,5 & & 8,4 & 1,2 \\
\hline Realiza atividade Física & & & 06 & & \\
\hline $\operatorname{sim}$ & 12,4 & 2,1 & & 9,9 & 1,9 \\
\hline Não & 12,1 & 1,8 & & 9,2 & 2,1 \\
\hline Tabagismo & & & 778 & & \\
\hline $\operatorname{Sim}$ & 11,9 & 2,1 & & 9,3 & 2,3 \\
\hline Não & 12,3 & 1,9 & & 9,5 & 2,0 \\
\hline Autopercepção da alimentação & & & 183 & & \\
\hline Positiva & 12 & 1,9 & & 9,4 & 2,0 \\
\hline Negativa & 12,4 & 2,0 & & 9,5 & 2,1 \\
\hline Comorbidade autorreferida & & & 157 & & \\
\hline $\operatorname{Sim}$ & 12 & 1,9 & & 9,6 & 2,0 \\
\hline Não & 12,4 & 2,0 & & 9,4 & 2,1 \\
\hline Uso de medicamento contínuo & & & 392 & & \\
\hline $\operatorname{Sim}$ & 12,1 & 2,0 & & 9,3 & 2,1 \\
\hline Não & 12,3 & 1,9 & & 9,6 & 2,0 \\
\hline Dor autorreferida & & & 25 & & \\
\hline $\operatorname{Sim}$ & 11,9 & 1,7 & & 9,4 & 2,0 \\
\hline Não & 12,6 & 2,1 & & 9,6 & 2,1 \\
\hline Dor autorreferida relacionada ao trabalho & & & 158 & & \\
\hline $\operatorname{sim}$ & 11,9 & 1,8 & & 9,3 & 2,0 \\
\hline Não & 12,4 & 2,0 & & 9,6 & 2,1 \\
\hline Satisfeito com trabalho & & & 88 & & \\
\hline Satisfeito & 12,6 & 1,9 & & 10,2 & 1,7 \\
\hline Insatisfeito & 12,0 & 1,9 & & 9,1 & 1,1 \\
\hline Afastamento do trabalho devido à dor & & & 748 & & \\
\hline $\operatorname{sim}$ & 12,2 & 1,4 & & 9,3 & 2,1 \\
\hline Não & 12,2 & 2,1 & & 9,5 & 2,0 \\
\hline
\end{tabular}

*DP = Desvio-Padrão. ${ }^{* *} \mathrm{APS}=$ Atenção Primária à Saúde. $\quad{ }^{* \star *} \mathrm{SM}=$ Salário Mínimo

\#QWLQ-bref = Quality of Working Life Questionnaire - versão abreviada.

Com relação ao domínio físico, constatou-se significância estatística para variável presença de dor $(\mathrm{p}=0,025)$, com destaque de maior média entre os profissionais que não apresen-

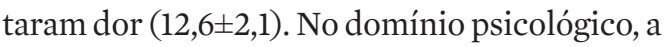
variável satisfação com o trabalho apresentou significância estatística $(\mathrm{p}=0,004)$, com maior média para aqueles que se encontram satisfeitos com o trabalho $(10,2 \pm 1,7)$.

A respeito do domínio pessoal, as variáveis que apresentaram significância estatística foram a cor da pele $(\mathrm{p}=0,012)$, com destaque 
para a cor branca, com média de $4,7 \pm 2,4$; a satisfação com o trabalho $(\mathrm{p}=0,012)$, com maior média entre os profissionais que afirmaram estar satisfeitos com o trabalho $(10,2 \pm 1,7)$. Os profissionais que referiram afastamento do trabalho $(9,5 \pm 2,0)$ também tiveram significância estatística $(\mathrm{p}=0,016)$.

No domínio profissional, as variáveis com significância estatística foram sexo $(\mathrm{p}=0,016)$, com destaque para as mulheres $(29,3 \pm 3,9)$; presença de dor $(\mathrm{p}=0,005)$, com maior média para aqueles que não relataram esse sintoma $(28,3 \pm 0,5)$; e a satisfação com o trabalho $(\mathrm{p}=0,005)$, com maior média para aqueles que se encontram satisfeitos $(28,8 \pm 4,8)$.
O escore total médio de estresse percebido pelos participantes foi de $24,5 \pm 6,0$, variando entre 10 e 40 pontos. Na tabela 3, está apresentada a associação entre nível de estresse percebido e variáveis sociodemográficas e de hábitos de vida dos participantes do estudo. As variáveis que se relacionaram com maior nível de estresse dos profissionais foram percepção quanto à alimentação $(\mathrm{p}=0,013)$, com destaque para aqueles que a consideram como não saudável $(25,9 \pm 5,8)$; à presença de dor $(p=0,002)$, com $26,3 \pm 6,0$; à dor relacionada ao trabalho ( $\mathrm{p}=0,004)$, com $26,3 \pm 5,9$; e à insatisfação com o trabalho $(\mathrm{p}=0,001)$, com $25,9 \pm 6,2$.

Tabela 3. Análise bivariada do Nível de Estresse Percebido (PSS-13) e variáveis sociodemográficas, ocupacionais e hábitos de vida dos profissionais da Atenção Primária à Saúde, distrito leste, Foz do Iguaçu (PR), 2018

\begin{tabular}{|c|c|c|c|}
\hline \multirow[b]{2}{*}{ Variáveis } & \multicolumn{3}{|c|}{ Escore total PSS-13* } \\
\hline & Média & $D P^{\star \star}$ & $\mathrm{p}$ \\
\hline Sexo & & & 0,088 \\
\hline Feminino & 24,9 & 6,2 & \\
\hline Masculino & 22,7 & 4,6 & \\
\hline Idade & & & 0,562 \\
\hline$>45$ anos & 24,9 & 6,2 & \\
\hline$\leq 45$ anos & 24,1 & 5,7 & \\
\hline Estado Civil & & & 0,762 \\
\hline Sem companheiro & 24,3 & 6,3 & \\
\hline Com companheiro & 24,8 & 5,7 & \\
\hline Cor da pele & & & 0,153 \\
\hline Não Branca & 23,9 & 6,2 & \\
\hline Branca & 25,5 & 5,6 & \\
\hline № de Filhos & & & 0,836 \\
\hline$\leq 2$ & 24,4 & 6,1 & \\
\hline$>2$ & 24,8 & 5,6 & \\
\hline Nível de formação & & & 0,608 \\
\hline Fundamental/Médio & 24,6 & 5,9 & \\
\hline Superior & 24,1 & 6,4 & \\
\hline Tempo Serviço na APS ${ }^{\star \star}$ & & & 0,088 \\
\hline$\leq 11$ anos & 23,7 & 6,5 & \\
\hline$>11$ anos & 25,2 & 5,6 & \\
\hline Renda familiar & & & 0,143 \\
\hline Até 3 SM\# & 21,7 & 5,1 & \\
\hline 04 a 07 SM & 25,1 & 5,4 & \\
\hline 08 ou mais SM & 24,0 & 8,1 & \\
\hline Tipo de moradia & & & 0,396 \\
\hline Própria & 24,2 & 5,6 & \\
\hline Alugada/cedida & 25,7 & 7,5 & \\
\hline
\end{tabular}


Tabela 3. (cont.)

\begin{tabular}{|c|c|c|c|}
\hline \multirow[b]{2}{*}{ Variáveis } & \multicolumn{2}{|c|}{ Escore total PSS-13* } & \multirow[b]{2}{*}{$\mathrm{p}$} \\
\hline & Média & $D P^{\star \star}$ & \\
\hline Realiza atividade Física & & & 0,051 \\
\hline Sim & 23,1 & 5,4 & \\
\hline Não & 25,4 & 6,2 & \\
\hline Tabagismo & & & 0,962 \\
\hline Sim & 24,4 & 7,5 & \\
\hline Não & 24,5 & 5,8 & \\
\hline Autopercepção da alimentação & & & 0,013 \\
\hline Positiva & 23,0 & 5,8 & \\
\hline Negativa & 25,9 & 5,8 & \\
\hline Comorbidade autorreferida & & & 0,065 \\
\hline Sim & 25,7 & 5,8 & \\
\hline Não & 23,4 & 6,0 & \\
\hline Uso de medicamento contínuo & & & 0,269 \\
\hline Sim & 25,1 & 6,3 & \\
\hline Não & 23,9 & 5,7 & \\
\hline Dor autorreferida & & & 0,002 \\
\hline $\operatorname{Sim}$ & 26,3 & 6,0 & \\
\hline Não & 22,7 & 5,3 & \\
\hline Dor autorreferida relacionada ao trabalho & & & 0,004 \\
\hline $\operatorname{Sim}$ & 26,3 & 5,9 & \\
\hline Não & 23,2 & 5,7 & \\
\hline Satisfeito com trabalho & & & $<0,001$ \\
\hline Satisfeito & 22,1 & 4,9 & \\
\hline Insatisfeito & 25,9 & 6,2 & \\
\hline Afastamento do trabalho devido à dor & & & 0,051 \\
\hline Sim & 26,2 & 5,0 & \\
\hline Não & 24,0 & 6,2 & \\
\hline
\end{tabular}

*PSS-13 = Escala de Estresse Percebido 13. ${ }^{* \star D P P}=$ Desvio-Padrão. ${ }^{\star *}$ APS $=$ Atenção Primária à Saúde \#SM = Salário Mínimo.

No modelo final de regressão linear múltipla (tabela 4) da QVT definido para o escore total constou a variável satisfação com trabalho $\left(\beta=45,65 ; \mathrm{IC}_{95} \%=[1,21 ; 1.728,64] ; \mathrm{p}=0,039\right)$; para o domínio psicológico, satisfação com trabalho $\left(\beta=2,67 ; \mathrm{IC}_{95} \%=[1,25 ; 5,70] ; \mathrm{p}=0,011\right)$; para o domínio pessoal, cor da pele $(\beta=3,01$;
$\left.\mathrm{IC}_{95} \%=[1,27 ; 7,15] ; \mathrm{p}=0,012\right) ;$ para o domínio profissional, $\operatorname{sexo}\left(\beta=0,10 ; \mathrm{IC}_{95} \%=[0,01 ; 0,99]\right.$; $\mathrm{p}=0,049)$. Com relação ao nível de estresse, $o$ modelo ficou definido pela variável satisfação com o trabalho $\left(\beta=0,06 ; \mathrm{IC}_{95} \%=[0,01 ; 0,54]\right.$; $\mathrm{p}=0,012$ ). 
Tabela 4. Fatores associados à qualidade de vida no trabalho e estresse percebido em profissionais da Atenção Primária à Saúde (análise múltipla), distrito leste, Foz do Iguaçu (PR), 2018

\begin{tabular}{|c|c|c|c|c|}
\hline \multirow{2}{*}{$\begin{array}{l}{ }^{\star} \text { QWLQ-bref } \\
\text { Domínio/Variável }\end{array}$} & \multicolumn{4}{|c|}{ IC95\% } \\
\hline & $\operatorname{Exp}(\beta)$ & Lim. Inf. & Lim. Sup. & p \\
\hline \multicolumn{5}{|l|}{ Psicológico } \\
\hline \multicolumn{5}{|c|}{ Satisfação com trabalho } \\
\hline Sim & 2,67 & 1,25 & 5,70 & 0,011 \\
\hline Não & 1 & & & \\
\hline \multicolumn{5}{|l|}{ Pessoal } \\
\hline \multicolumn{5}{|l|}{ Cor da Pele } \\
\hline Branca & 3,01 & 1,27 & 7,15 & 0,012 \\
\hline Não Branca & 1 & & & \\
\hline \multicolumn{5}{|l|}{ Profissional } \\
\hline \multicolumn{5}{|l|}{ Sexo } \\
\hline Feminino & 0,10 & 0,01 & 0,99 & 0,049 \\
\hline Masculino & 1 & & & \\
\hline \multicolumn{5}{|l|}{ Escore Global } \\
\hline \multicolumn{5}{|c|}{ Satisfação com trabalho } \\
\hline Sim & 45,65 & 1,21 & 1728,64 & 0,039 \\
\hline Não & 1 & & & \\
\hline **PSS-13 & & IC95\% & & \\
\hline Variável & $\operatorname{Exp}(\beta)$ & Lim. Inf. & Lim. Sup. & $\mathbf{P}$ \\
\hline \multicolumn{5}{|c|}{ Satisfação com trabalho } \\
\hline Sim & 0,06 & 0,01 & 0,54 & 0,012 \\
\hline Não & 1 & & & \\
\hline
\end{tabular}

${ }^{\star}$ QWLQ-bref = Quality of Working Life Questionnaire - versão abreviada. ${ }^{\star * P S S-13=~ E s c a l a ~ d e ~ E s t r e s s e ~ P e r c e b i d o ~} 13$.

\section{Discussão}

Neste estudo, a avaliação da qualidade de vida e do nível de estresse utilizando-se de instrumentos validados permitiu aferir aspectos importantes que marcam a vida do profissional de saúde no trabalho na APS. Os achados do estudo apontaram satisfatória QVT e níveis médios de estresse entre os participantes. Além disso, mostrou que a satisfação com o trabalho está associada à QVT global e ao nível de estresse dos trabalhadores da APS. Outras variáveis, como a cor da pele e o sexo, estão associadas aos domínios pessoal e profissional da QVT, respectivamente.
A associação significativa da satisfação com o trabalho com o escore total de QVT e, também, especificamente, com o domínio psicológico revela que os profissionais mais satisfeitos apresentaram maior qualidade de vida relacionada ao trabalho neste estudo. A satisfação com o trabalho é um indicador muito útil na avaliação da QVT e está relacionada a estados internos do indivíduo e de grupos que predizem elementos da organização do trabalho em si ${ }^{\mathbf{1 4}}$. Além disso, essa variável tem sido pesquisada em muitos estudos que apontam que aqueles profissionais que se consideram mais satisfeitos apresentam menores chances de desenvolver síndrome de burnout ${ }^{\mathbf{1 5 , 1 6}}$, são 
menos vulneráveis a riscos psicossociais ${ }^{16} \mathrm{e}$ possuem maior frequência na autopercepção positiva do estado de saúde ${ }^{\mathbf{1 7}}$.

Considerando a satisfação no trabalho, alguns fatores têm sido descritos como determinantes, tais como satisfação com o trabalho executado; possibilidade de futuro na organização; reconhecimento pelos resultados alcançados; salário percebido; benefícios auferidos; relacionamento humano na equipe e na organização; ambiente psicológico e físico de trabalho; liberdade de atuar e responsabilidade de tomar decisões; possibilidade de estar engajado e de participar ativamente ${ }^{\mathbf{1 8 - 2 0}}$.

Pesquisa com médicos de Organização Social de Saúde que presta assistência ao SUS evidenciou que a satisfação com o ambiente de trabalho está relacionada com a equipe de trabalho, gerência da unidade e reconhecimento profissional ${ }^{21}$. Nessa perspectiva, quando se discute a satisfação com o trabalho, existem muitos fatores a serem considerados na realidade da APS investigada neste estudo, que podem se refletir no desempenho profissional frente às demandas dos usuários de saúde.

No domínio psicológico, evidenciou-se que trabalhadores da APS que são satisfeitos com o trabalho possuem escore 2,67 vezes maior que os trabalhadores que não estão satisfeitos. Esse domínio da QVT envolve aspectos de satisfação pessoal, motivação no trabalho e autoestima dos trabalhadores. É possível que os profissionais da APS que se consideram satisfeitos com o trabalho também apresentem satisfação pessoal, além de serem motivados no trabalho e possuírem uma elevada autoestima.

A análise da QVT mostrou também, nesta investigação, que o domínio pessoal referente a aspectos familiares, crenças pessoais, crenças religiosas e aspectos culturais que podem influenciar o trabalho ${ }^{12}$ foi associado à cor da pele. Os trabalhadores que possuem a cor da pele branca possuem escore 3,01 vezes maior que os trabalhadores de cor não branca no domínio pessoal. Pelo fato de o estudo ter sido realizado em município da região Sul do Brasil, destaca-se o alinhamento com outras pesquisas com trabalhadores da área da saúde nessa região que apresentam maior contingente de pessoas de cor branca ${ }^{22-24}$. Acredita-se que a adaptação religiosa, os aspectos familiares, pessoais e culturais sejam mais facilitados pelas pessoas de cor branca neste estudo devido à elevada distribuição desse público nessa região, fazendo com que tenham melhor QVT no domínio pessoal. Não foram encontrados estudos na literatura que também apontem que a cor da pele influencie a QVT.

Nesta investigação, o sexo esteve associado ao domínio profissional, o qual considera os aspectos e as atitudes organizacionais que influenciem a QVT dos profissionais. A análise desse domínio revela que não depende apenas dos trabalhadores em si para haver melhorias na QVT, mas das condições de trabalho oferecidas pelas empresas, dos benefícios e da sua responsabilidade social ${ }^{12}$. É interessante ressaltar a associação desse domínio com a variável sexo, o que indica que é um fator pessoal importante que influencia na melhor ou pior QVT com relação às atitudes e aos aspectos organizacionais do trabalho em saúde. Neste estudo, indivíduos do sexo masculino tiveram escore $90,0 \%$ maior que o feminino no domínio profissional, o que aponta que ser homem pode ser um fator de proteção para a QVT nesse domínio.

O sexo tem sido discutido na literatura com destaque para algumas características relacionadas às mulheres ${ }^{24-26}$. A predominância de mulheres nesta investigação pode apontar para a maior participação feminina no mercado de trabalho na nossa sociedade e o fato de elas se identificarem com o trabalho em saúde, que muitas vezes está relacionado ao cuidado com o outro ${ }^{25}$. Entretanto, a inserção da mulher pode gerar vulnerabilidade, devido à discriminação de gênero e à possível distribuição injusta de tarefas ${ }^{27}$. Ressalta-se, ainda, que muitas vezes a dupla jornada de trabalho adotada concilia o cuidado da família e da casa com o serviço em $\mathrm{si}^{\mathbf{2 5 , 2 6}}$. A alta sobrecarga doméstica também tem sido associada com a autoavaliação negativa do estado de saúde em 
estudo com profissionais da APS no estado da Bahia ${ }^{17}$. Acredita-se que o acúmulo de atividades pela mulher pode estar interferindo no menor escore do domínio profissional, fazendo com que ela perceba menos aspectos positivos e atitudes organizacionais do trabalho em saúde, contribuindo para uma menor QVT no domínio profissional.

Diferentemente dos resultados encontrados no presente estudo, uma investigação realizada nas UBS de Santa Maria (RS) encontrou que profissionais do sexo feminino e mais jovens apresentaram maior QVT. Tal pesquisa ressaltou que os profissionais apresentaram desgastes físicos, emocionais e pouca adesão a comportamentos saudáveis, fato que está atrelado à sua rotina de trabalho $\mathbf{2 4}$.

Com relação ao nível de estresse pesquisado, esta pesquisa revelou que aqueles profissionais que não estão satisfeitos com o trabalho têm 94,0\% maior escore de estresse percebido do que aqueles que estão satisfeitos. Os profissionais que estão satisfeitos com o trabalho apresentam menores níveis de estresse, representando um fator de proteção relevante no contexto laboral.

Entre os fatores causadores de insatisfação no trabalho em saúde, bem como no âmbito da APS, encontra-se o trabalhar em local que não é de sua escolha, a sobrecarga de trabalho e a violência, que têm sido responsáveis por doenças relacionadas ao trabalho, absenteísmo e problemas psicossociais ${ }^{28}$. Essas questões podem ocasionar agravos, como estresse, ansiedade, depressão, outros transtornos mentais e abuso de álcool e drogas ${ }^{29}$.

Os níveis elevados de estresse predispõem os profissionais à síndrome de burnout. $\mathrm{O}$ trabalho na APS faz com que os profissionais estejam mais próximos da comunidade e de suas realidades, sentindo-se cobrados e pressionados a dar respostas às necessidades das comunidades que atendem ${ }^{\mathbf{8}, 30}$. Além disso, são cobrados por produtividade num cenário com carência de recursos humanos, materiais e de infraestrutura e com visão curativista que diverge dos ideais da APS, o que demonstra ser um trabalho complexo por exigir a utilização da tecnologia leve 7,31 .

Pesquisa de revisão integrativa da literatura identificou que os trabalhadores de saúde da APS apresentam-se esgotados devido às inadequadas condições de trabalho, caracterizadas por escassez de recursos humanos e físicos, que levam a sobrecarga de trabalho, violência no ambiente de trabalho e dificuldade no trabalho em equipe, apesar de apresentarem-se satisfeitos com o ambiente de trabalho ${ }^{\mathbf{3 2}}$.

O estresse contínuo no trabalho pode trazer consequências prejudiciais à saúde mental e física do trabalhador, tais como o desenvolvimento da síndrome metabólica, de distúrbios do sono, diabetes, hipertensão, enfermidades psicossomáticas, síndrome de burnout, depressão, uso de substâncias psicoativas, além de queda na produtividade, absenteísmo, insatisfação laboral e baixa QVT ${ }^{3,33}$.

O estudo ora apresentado revela em sua estrutura algumas limitações dignas de nota. Primeiramente, o fato relacionado ao tipo de delineamento conduzido, que foi o transversal, que impede que os resultados apresentados sejam tomados como causa e efeito, devido a não aferição da temporalidade. Outro ponto importante foram os aspectos que envolveram a amostra utilizada de um distrito sanitário do município, o que não pode ser generalizado para outras realidades. Apesar de tais limitações, sob a perspectiva dos instrumentos avaliativos empregados, os resultados desta investigação são potencialmente relevantes, pois foram obtidos a partir de uma escala com capacidade de inferir consequências clínicas oriundas de níveis de estresse, além da repercussão na QVT dos profissionais. Essa investigação pôde contribuir para revelar um contexto até então desconhecido, que é similar ao de outras localidades da conjuntura laboral da APS no País.

Foram utilizados diferentes instrumentos e escalas de autorrelato, visando a aumentar a confiabilidade do estudo para avaliação de qualidade de vida e estresse laboral dos trabalhadores. Apesar disso, este estudo abordou 
um leque de variáveis que potencialmente influenciam a QVT e os níveis de estresse, destacando aspectos que podem ser utilizados por gestores e por profissionais da saúde. Destaca-se, ainda, que foi possível desvelar o atual panorama da QVT e os níveis de estresse dos profissionais da APS, o que até então não havia sido feito em investigações anteriores.

\section{Conclusões}

Conclui-se que a satisfação com o trabalho constitui importante fator na determinação da melhor QVT e no domínio psicológico, bem como na proteção contra o estresse. Outros fatores relacionados à melhor QVT nos domínios pesquisados foram: a cor da pele branca no domínio pessoal e o sexo masculino no domínio profissional. Esses resultados devem ser considerados pelos serviços de saúde, bem como na formulação de políticas públicas na abordagem à saúde do trabalhador, especificamente na APS. Ao se reconhecer a satisfação com o trabalho como importante fator protetor da QVT e da percepção do estresse, poderão ser feitas intervenções oportunas para melhoria da saúde do trabalhador. Sugere-se, também, a formação de grupos para discutir os mecanismos de enfrentamento do estresse, a fim de que sejam adotados em momentos oportunos, permitindo, então, um manejo adequado dos estressores, a consequente prevenção do estresse laboral e a melhora na QVT. Por fim, é importante ressaltar a necessidade de realizar estudos longitudinais que permitam verificar a associação causal entre QVT, nível de estresse dos profissionais de saúde e seus fatores associados.

\section{Colaboradores}

Lima GKM (0000-0003-2378-1404)* contribuiu para a concepção, planejamento e escrita do manuscrito. Gomes LMX (0000-00016442-5719)* contribuiu significativamente para a interpretação dos dados, revisão crítica e final do conteúdo do manuscrito. E Barbosa TLA (0000-0002-6985-9548)* contribuiu para concepção, planejamento, análise estatística, interpretação de dados e revisão crítica do conteúdo do manuscrito. 


\section{Referências}

1. Brasil. Decreto $\mathrm{n}^{0} 7.602$, de 07 de novembro de 2011. Dispõe sobre a Política de Segurança e Saúde no Trabalho - PNSST. Diário Oficial da União, Brasília, DF, [internet]. 7 Nov 2011 [acesso em 2019 jun 20]. Disponível em: http://www.planalto.gov.br/ccivil_03/_ ato2011-2014/2011/decreto/d7602.htm.

2. Ferreira MC. Qualidade de vida no trabalho (qvt): do assistencialismo à promoção efetiva. [internet]. 2015 [acesso em 2019 jun 23]; 11(2):28-35. Disponível em: http://www.laboreal.up.pt/files/articles/28_35_l.pdf.

3. Aquino AS, Fernandes ACP. Quality of life and work. J Health Sci Insti [internet]. 2013 [acesso em 2019 abr 12]; 31(1):53-58. Disponível em: https://www. unip.br/presencial/comunicacao/publicacoes/ics/ edicoes/2013/01_jan-mar/V31_n1_2013_p53a58.pdf.

4. Ribeiro LA, Santana LC. Qualidade de vida no trabalho: fator decisivo para o sucesso organizacional. RIC [internet]. 2015 [acesso em 2019 maio 20]; 2(2):75-96. Disponível em: https://www.cairu.br/riccairu/pdf/ artigos/2/06_QUALIDADE_VIDA_TRABALHO.pdf.

5. Brasil. Ministério da Saúde. Portaria n ${ }^{0} 2.436$, de 21 de setembro de 2017. Aprova a Política Nacional de Atenção Básica, estabelecendo a revisão de diretrizes para a organização da Atenção Básica, no âmbito do Sistema Único de Saúde (SUS). Brasília, DF: Ministério da Saúde; [internet] 2017. [acesso em 2019 mar 22]. Disponível em: https://bvsms.saude.gov.br/bvs/ saudelegis/gm/2017/prt2436_22_09_2017.html.

6. Martins MB, Araújo TPF, Ferreira LB, et al. Qualidade de vida dos enfermeiros da atenção primária à saúde de Brasília - DF. Rev. Eletrônica Gestão e Saúde [internet]. 2013 [acesso em 2019 fev 7]; 4(2):293302. Disponível em: https://periodicos.unb.br/index. php/rgs/article/view/249/238.

7. Maissiat GS, Lautert L, Pai DD, et al. Contexto de trabalho, prazer e sofrimento na atenção básica em saúde. Rev. Gaúcha Enferm [internet]. 2015 [acesso em 2019 fev 23]; 36(2):42-49. Disponível em: ht- tps://www.scielo.br/pdf/rgenf/v36n2/pt_1983-1447rgenf-36-02-00042.pdf.

8. Lima AS, Farah BF, Bustamante-Teixeira MT. Análise da prevalência da síndrome de Burnout em profissionais da atenção primária em saúde. Trab. educ. saúde [internet]. 2018 [acesso em 2019 fev 14]; 16(1):283303. Disponível em: https://www.scielo.br/pdf/tes/ v16n1/1678-1007-tes-1981-7746-sol00099.pdf.

9. Teles MAB, Barbosa MR, Vargas AMD, et al. Psychosocial work conditions and quality of life among primary health care employees: a cross sectional study. Health and Quality of Life Outcomes [internet]. 2014 [acesso em 2019 mar 10]; 12:72. Disponível em: https:// hqlo.biomedcentral.com/track/pdf/10.1186/14777525-12-72.

10. Azevedo BS, Nery AA, Cardoso JP. Estresse ocupacional e insatisfação com a qualidade de vida no trabalho da enfermagem. Texto Contexto Enferm [internet]. 2017 [acesso em 2019 mar 8]; 26(1):e3940015. Disponível em: https://www.scielo.br/pdf/tce/v26nl/ pt_1980-265X-tce-26-01-e3940015.pdf.

11. Instituto Brasileiro de Geografia e Estatística. Estimativas da população. Brasília, DF: IBGE [internet]. 2018. [acesso em $2019 \mathrm{fev}$ 10]. Disponível em: https://www.ibge.gov.br/estatisticas/sociais/ populacao/9103-estimativas-de-populacao.html?t= resultados $\% 2527 \% 2527 \% 2527$.

12. Cheremeta M, Pedroso B, Pilatti LA, et al. Construção da versão abreviada do QWLQ-78: um instrumento de avaliação da qualidade de vida no trabalho. Revista Brasileira de Qualidade de Vida [internet]. 2011 [acesso em 2019 jan 15]; 3(1):1-15. Disponível em: https:// periodicos.utfpr.edu.br/rbqv/article/view/758/600.

13. Cohen S, Karmack T, Mermelsteinm R. A global measure of perceived stress. J Health Soc Behav [internet]. 1983 [acesso em 2019 fev 22]; 24(4):385-96. Disponível em: https://www-jstor-org.ez27.periodicos.capes.gov.br/stable/pdf/2136404.pdf?ab_ 
segments $=0 \% 252 \mathrm{Fbasic} \_$SYC $-5187 \% 252 \mathrm{Ftest} \& \mathrm{refr}$ eqid=excelsior\%3A0e780a3f5bloc574clc083161f33 $2 a a b$.

14. Sampaio JR. Qualidade de vida no trabalho: perspectivas e desafios atuais. Rev. psicol. organ. Trab [internet]. 2012 [acesso em 2019 abr 12]; 12(1):121-136. Disponível em: http://pepsic.bvsalud.org/pdf/rpot/ v12nl/v12nlall.pdf.

15. Navarro-González D, Ayechu-Díaz A, Huarte-Labiano I. Prevalencia del síndrome del Burnout y factores asociados a dicho síndrome en los profesionales sanitarios de atención primaria. Semergen [internet]. 2015 [acesso em 2019 mar 19]; 41(4):191-198. Disponível em: https://www.elsevier.es/es-revista-medicina-familia-semergen-40-pdf-S1138359314001270.

16. Merino-Plaza MJ, Carrera-Hueso FJ, Arribas-Boscá $\mathrm{N}$, et al. Burnout y factores de riesgo psicosocial en el personal de un hospital de larga estancia. Cad. Saúde Pública [internet]. 2018 [acesso em 2020 jul 8]; 34(11):e00189217. Disponível em: https://www.scielo. br/pdf/csp/v34nll/1678-4464-csp-34-11-e00189217.pdf.

17. Lua I, Almeida MMG, Araújo TM, et al. Autoavaliação negativa da saúde em trabalhadoras de enfermagem da atenção básica. Trab educ saúde [internet]. 2018 [acesso em 2019 ago 20]; 16(3):1301-1319. Disponível em: https://www.scielo.br/pdf/tes/v16n3/1678-1007tes-16-03-1301.pdf.

18. Chiavenato I. Gestão de pessoas: o novo papel dos recursos humanos nas organizações. 4. ed. Barueri: Manole;2014.

19. Lu Y, Hu XM, Huang XL, et al. Job satisfaction and associated factors among healthcare staff: a cross-sectional study in Guangdong Province, China. BMJ Open [internet]. 2016 [acesso em 2019 jul 9]; 6(7):e011388. Disponível em: https://bmjopen.bmj.com/content/ bmjopen/6/7/e011388.full.pdf.

20. Collins S. Statutory social workers: stress, job satisfaction, coping, social support and individual differences. Br. J. Soc. Work [internet]. 2008 [acesso em 2019 maio 19]; 38(6):1173-1193. Disponível em: ht- tps://academic-oup-com.ez27.periodicos.capes.gov. br/bjsw/article/38/6/1173/1660225.

21. Pegorari F, Morgado F, Alves BCA, et al. Nível de satisfação dos profissionais médicos quanto ao seu ambiente de trabalho em organização social de saúde de assistência ao sistema único de saúde do Brasil. J Hum Growth Dev [internet]. 2017 [acesso em 2019 fev 11]; 27(3):322-331. Disponível em: http://pepsic. bvsalud.org/pdf/rbcdh/v27n3/pt_10.pdf.

22. Raffone AM, Hennington EA. Avaliação da capacidade funcional dos trabalhadores de enfermagem. Rev. Saúde Públ [internet]. 2005 [acesso em 2019 maio 13]; 39(4):669-676. Disponível em: https://www.scielo.br/ pdf/rsp/v39n4/25542.pdf.

23. Magnago TSBS, Lima ACS, Prochnow A, et al. Intensidade da dor musculoesquelética e a (in) capacidade para o trabalho na enfermagem. Rev. Latino-Am. Enfermagem [internet]. 2012 [acesso em 2019 maio 18]; 20(6):1125-1133. Disponível em: https://www.scielo. br/pdf/rlae/v20n6/pt_15.pdf.

24. Medeiros PA, Silva LC, Amarante IM, et al. Condições de saúde entre profissionais da atenção básica em saúde do município de Santa Maria - RS. RBCS [internet]. 2016 [acesso em 2019 abr 24]; 20(2):115122. Disponível em: https://periodicos.ufpb.br/ojs2/ index.php/rbcs/article/view/18961/15739.

25. Santos FAAS, Sousa LP, Serra MAAO, et al. Fatores que influenciam na qualidade de vida dos agentes comunitários de saúde. Acta paulista de enfermagem [internet]. 2016 [acesso em 2019 mar 22]; 29(2):191197. Disponível em: https://www.scielo.br/pdf/ape/ v29n2/1982-0194-ape-29-02-0191.pdf.

26. Tavakoli-Fard N, Mortazavi SA, Kuhpayehzadeh J, et al. Quality of life, work ability and other important indicators of women's occupational health. Int J Occup Med Environ Health [internet]. 2016 [acesso em 2019 abr 11]; 29(1):77-84. Disponível em: http://ijomeh.eu/ Quality-of-life-work-ability-and-other-important-indicators-of-women-s-health-worker,58504,0,2.html.

27. Landsbergis PA, Dobson M, Koutsouras G, et al. Job 
strain and Ambulatory Blood Pressure: a meta-analysis and systematic review. Am J Public Health [internet]. 2013 [acesso em 2019 jun 17]; 103(3):e61-e71. Disponível em: https://www.ncbi.nlm.nih.gov/pmc/ articles/PMC3673518/pdf/AJPH.2012.301153.pdf.

28. Munyewende PO, Rispel LC, Chirwa T. Positive practice environments influence job satisfaction of primary health care clinic nursing managers in two South African Provinces. Human Resources for Health [internet]. 2014 [acesso em 2019 jun 2]; 12:27. Disponível em: https://www.ncbi.nlm.nih.gov/pmc/articles/ PMC4024627/pdf/1478-4491-12-27.pdf.

29. Fernandes MA. Riscos ocupacionais e o adoecimento de trabalhadores de saúde de um hospital psiquiátrico do Piauí. [tese]: Ribeirão Preto: Escola de Enfermagem de Ribeirão Preto, Universidade de São Paulo; 2014. 130 p. [acesso em 2020 set 4]. Disponível em: https://www.teses.usp.br/teses/disponiveis/22/22132/tde-07012015-143853/pt-br.php.

30. Silva SCPS, Nunes MAP, Santana VR, et al. A síndrome de burnout em profissionais da rede de atenção primária à saúde de Aracaju, Brasil. Ciênc. Saúde Colet. [internet]. 2015 [acesso em 2019 maio 29];
20(10):3011-3020. Disponível em: https://www.scielo.br/pdf/csc/v20n10/1413-8123-csc-20-10-3011.pdf.

31. Ribeiro RP, Marziale MHP, Martins JT, et al. Estresse ocupacional entre trabalhadores de saúde de um hospital universitário. Rev Gaúcha Enferm [internet]. 2018 [acesso em 2019 jul 15]; 39:65127. Disponível em: https://www.scielo.br/pdf/rgenf/v39/19831447-rgenf-39-e65127.pdf.

32. Garcia GPA, Marziale MHP. Indicators of burnout in Primary Health Care workers. Rev Bras Enferm [internet]. 2018 [acesso em 2019 abr 20]; 71(supl5):23342342. Disponível em: https://www.scielo.br/pdf/reben/v71s5/pt_0034-7167-reben-71-s5-2334.pdf.

33. Moura A, Lunardi R, Volpato R, et al. Fatores associados à ansiedade entre profissionais da atenção básica. Revista Portuguesa de Enfermagem de Saúde Mental [internet]. 2018 [acesso em 2019 jun 13]; (19):17-26. Disponível em: http://www.scielo.mec.pt/ pdf/rpesm/n19/n19a03.pdf.

Recebido em 26/12/2019

Aprovado em 14/05/2020

Conflito de interesses: inexistente

Suporte financeiro: não houve 